\title{
Frontières
}

\section{Mieux comprendre pour mieux intervenir auprès du jeune suicidant}

\section{Réal Labelle et Richard Boyer}

Volume 12, numéro 1, automne 1999

Suicides, générations et culture

URI : https://id.erudit.org/iderudit/1074513ar

DOI : https://doi.org/10.7202/1074513ar

Aller au sommaire du numéro

Éditeur(s)

Université du Québec à Montréal

ISSN

1180-3479 (imprimé)

1916-0976 (numérique)

Découvrir la revue

Citer cet article

Labelle, R. \& Boyer, R. (1999). Mieux comprendre pour mieux intervenir auprès du jeune suicidant. Frontières, 12(1), 79-83. https://doi.org/10.7202/1074513ar
Résumé de l'article

Comme intervenant professionnel, quelle aide convient-il d'offrir au jeune suicidant consultant? Des résultats d'une étude exploratoire sont d'abord présentés pour illustrer les propos de vingt-quatre jeunes quant à leur demande d'aide formelle (contexte, recours et satisfaction) avant de faire leur tentative de suicide. Ces résultats sont ensuite discutés de manière à soulever divers enjeux d'intervention. Cette réflexion suggère qu'il est important pour l'intervenant : (1) d'accueillir et d'évaluer l'angoisse du jeune, (2) de cerner ses capacités d'intervention, (3) d'adopter envers le jeune une approche personnalisée et circonscrite et (4) d'être autocritique vis-à-vis l'aide à dispenser et la psychosociologie du jeune. 


\section{Résumé}

Comme intervenant professionnel, quelle aide convient-il d'offrir au jeune suicidant consultant? Des résultats d'une étude exploratoire sont d'abord présentés pour illustrer les propos de vingt-quatre jeunes quant à leur demande d'aide formelle (contexte, recours et satisfaction) avant de faire leur tentative de suicide. Ces résultats sont ensuite discutés de manière à soulever divers enjeux d'intervention. Cette réflexion suggère qu'il est important pour l'intervenant: (1) d'accueillir et d'évaluer l'angoisse du jeune, (2) de cerner ses capacités d'intervention, (3) d'adopter envers le jeune une approche personnalisée et circonscrite et (4) d'être autocritique vis-à-vis l'aide à dispenser et la psychosociologie du jeune.

Mots clés: suicide - jeunesse - aide formelle intervention

\section{Abstract}

What is the appropriate assistance professional caregivers should provide to suicidal youths who seek help? Results from an exploratory study are first presented to illustrate what twenty-four young people had to say about their formal requests for help (environment, satisfaction, and the ability to turn to someone) before their first suicide attempt. These results are then discussed so as to raise various intervention issues. This consideration suggests that it is important for caregivers to (1) to be open and to evaluate young people's distress, (2) to determine their own abilities to intervene, (3) to adopt a personalized and controlled approach toward youths, and (4) to critique themselves as to the assistance they provide and to young people's psychosociology.

Keywords: suicide - youth - formal requests intervention

\section{Mieux comprendre pour mieux intervenir auprès du jeune suicidant'}

«IL N'A PAS VU, SUR MON VISAGE, TOUT MON DÉSESPOIR [...]. C'EST DE ÇA QUE J'AVAIS LE PLUS BESOIN À CE MOMENT-LÀ. QUELQU'UN QUI CONSTATE MA DOULEUR ET QUI ME VIENNE EN AIDE [...]. PARCE QUE, EN Y REPENSANT, JE N'AVAIS PLUS DE FORCE, J'ÉTAIS VIDÉ ET JE N'AVAIS AUCUNE ÉNERGIE POUR EXPRIMER TOUT MON DÉGOÛT FACE À CETTE VIE INSENSÉE [...]».

\author{
Réal Labelle, \\ professeur au Département de psychologie, UQTR, \\ membre du CRISE. \\ avec la collaboration de Richard Boyer, \\ chercheur au Centre de recherche Fernand-Seguin.
}

Il n'est pas toujours facile de bien cerner la réalité d'un jeune qui souffre, pour pouvoir l'aider. Les propos cités en exergue illustrent cet état de chose. L'histoire d'Adrien, un jeune homme de 21 ans qui, quelques jours après avoir formulé une demande d'aide formelle, a sauté d'un viaduc et s'est écrasé sur un terre-plein de ciment, démontre à quel point il peut être difficile pour un intervenant d'évaluer la souffrance d'un jeune qui appelle à l'aide. Par miracle, Adrien a survécu à sa chute et, dans une entrevue ultérieure, il nous a livré ses états d'âme relativement à sa rencontre avec un aidant professionnel.

$$
* * *
$$

Par définition, on entend par aide formelle l'aide privée, publique ou communautaire offerte par des intervenants qui ont reçu une formation. Au Québec, le comportement de recherche d'aide formelle a été décrit par Dulac ${ }^{2}$ et étudié chez les suicidants ${ }^{3}$ par Lavoie et al. ${ }^{4}$. Plus précisément, le premier auteur s'inspire du modèle de Gross et McMullen $^{5}$ pour exposer les trois étapes sous-jacentes à une démarche d'assistance. Il y aurait successivement la reconnaissance d'avoir un problème, la décision de s'en sortir avec l'appui d'autrui, et le recours aux services d'aide. La demande d'aide s'inscrit ainsi à la dernière phase du processus. Les seconds chercheurs examinent les relations existant entre un groupe de 128 suicidants et son réseau d'aide naturelle et formelle. Leurs résultats démontrent notamment que l'entourage est présent et offre un soutien réel, bien que ce soutien envers des suicidants qui ont des problèmes graves ou chroniques soit parfois malhabile. De plus, leurs observations indiquent que les professionnels de la santé et les intervenants communautaires occupent une place mineure dans le processus de recherche 
d'aide des suicidants enquêtés, exception faite des médecins généralistes.

Le présent texte cherche à mieux comprendre la réalité de jeunes suicidants québécois en demande d'aide afin de mieux intervenir auprès d'eux. Pour ce faire, le texte se divise en trois parties. Nous examinerons tout d'abord les résultats d'une étude exploratoire portant sur la demande d'aide formelle de jeunes suicidants québécois. Nous nous baserons ensuite sur ces résultats pour soulever divers enjeux à considérer en matière d'intervention auprès de ce groupe particulier. Enfin, nous conclurons en situant notre point de vue par rapport à la Stratégie québécoise d'action face au suicide 6 .

\section{ÉTUDE EXPLORATOIRE PORTANT SUR LA DEMANDE D'AIDE FORMELLE DE JEUNES SUICIDANTS}

En janvier 1995, nous avons accepté le mandat du Conseil permanent de la jeunesse ${ }^{7}$ de mener une étude exploratoire ${ }^{8}$ portant sur la demande d'aide formelle de jeunes suicidants. Le projet avait pour but de laisser la parole aux jeunes, de manière à mieux connaître le contexte, le recours et la satisfaction de ceux-ci face à l'aide demandée et reçue, et ce, quelque temps avant de faire une tentative de suicide. Comportant cinq étapes ${ }^{9}$, la méthode s'appuyait sur une analyse de cas multiples avec des analyses statistiques descriptives et des analyses de contenu du discours ${ }^{10}$. Le groupe des participants comprenait 24 jeunes francophones âgés entre 15 et 29 ans et ayant fait une demande d'aide formelle avant d'avoir fait une tentative de suicide récente ${ }^{11}$. Ces répondants, en majorité des femmes $(63 \%)$ et des jeunes aux études (58\%), résidaient dans 11 régions du Québec ${ }^{12}$. La première partie de ce texte présente les principaux résultats associés à ce travail.

\section{CONTEXTE ENTOURANT LA DEMANDE D'AIDE}

Le travail de reconstruction des circonstances entourant la demande d'aide formelle a permis d'accéder au matériel anecdotique suivant. Dans la grande majorité des cas, les jeunes suicidants font appel à des intervenants avant de faire leur tentative de suicide, parce qu'ils ont peur de «craquer». Cette crainte de perdre le contrôle se dénote par un discours qui traduit un passé lourd et un présent douloureux. En fait, plusieurs jeunes racontent avoir vécu, en bas âge, des épisodes de grand débordement affectif se manifestant par des crises de rage ou de tristesse, suite à des expériences très difficiles ${ }^{13}$. Pour plus de $63 \%$ d'entre eux, cet état était si douloureux qu'ils avaient tenté de se tuer ${ }^{14}$. Aujourd'hui, quelques mois ou quelques années plus tard, ces mêmes jeunes doivent affronter à nouveau des problèmes importants $^{15}$. Ils disent douter de leurs capacités personnelles pour composer avec ces événements et appréhendent la venue d'autres épisodes d'angoisse intense pouvant les amener à poser un geste désespéré. C'est dans ce contexte tourmenté qu'ils ont fait une demande d'aide formelle.

\section{RECOURS AUX SERVICES D'AIDE}

$\mathrm{Au}$ cours des dernières années, la désinstitutionnalisation des services en santé mentale a favorisé le développement de ressources issues d'une multitude d'organismes. La personne désespérée qui se trouve subitement aux prises avec des difficultés majeures peut recourir à une panoplie d'intervenants, allant du psychiatre de l'urgence de l'hôpital au psychologue de la clinique du CLSC, en passant par l'intervenant bénévole d'un centre d'écoute. Dans ces circonstances, où et dans quelle proportion ces jeunes adressent-ils leur demande d'aide? Dans 92 \% des cas, les jeunes rencontrés rapportent recourir à au moins un service d'aide formelle avant leur dernière tentative de suicide. Les services utilisés renvoient à huit ressources différentes. Le Tableau 1 présente le taux d'utilisation de chacun de ces services selon le sexe et l'ensemble des jeunes interviewés. Il importe de souligner l'usage fréquent des milieux scolaires, des hôpitaux et des centres d'écoute. Par ailleurs, il semble que les répondants féminins sollicitent plus sou- vent que les hommes l'aide dispensée par des organismes communautaires, par le CLSC et par des centres de prévention suicide.

\section{SATISFACTION FACE À L'AIDE REÇUE}

Le Tableau 1 présente aussi le degré de satisfaction des jeunes face à l'aide reçue $^{16}$. Paradoxalement, les ressources les moins appréciées, c'est-à-dire celles qui obtiennent une moyenne inférieure à sept, sont celles qui sont les plus utilisées (à l'exception de la DPJ). Comment expliquer ce résultat inattendu? Le rapport interne entre «recours» et «satisfaction» des services est difficile à cerner. Il est toutefois possible d'émettre deux hypothèses. La première porte sur la commodité et la proximité de l'aide obtenue. Plusieurs jeunes ont dit s'être tournés vers une ressource parce qu'elle était accessible et proche, et ce, sans égard à la spécificité de la ressource. À titre d'exemple, plusieurs élèves en mal de vivre ont sollicité du temps d'écoute et de parole de la part des infirmières de leur école. Or, ils ont reçu très peu d'aide de ce côté en raison des craintes excessives des intervenantes; en effet, nombre d'entre elles se sentaient obligées d'adopter une approche interventionniste afin d'éviter une récidive, oubliant du même coup de s'adresser à la souffrance des jeunes. La seconde hypothèse repose sur les caractéristiques psychologiques des répondants lors de l'entrevue. En effet, il s'avère que l'appréciation des ressources varie en fonction de la détresse psychologique et de la tendance à la désirabilité sociale des jeunes au moment de l'entretien. Le Tableau 2 illustre cette observation en regard des ressources les plus utilisées. Ainsi, plus un jeune est en détresse

\section{Tableau 1 \\ Recours et satisfaction des ressources utilisées selon le sexe et l'ensemble des jeunes}

\begin{tabular}{l|c|c|c|c|c|c} 
Ressources & \multicolumn{2}{|c|}{$\begin{array}{c}\text { Hommes } \\
\mathrm{n}=9\end{array}$} & \multicolumn{2}{c|}{$\begin{array}{c}\text { Femmes } \\
\mathrm{n}=15\end{array}$} & \multicolumn{2}{c}{ Total } \\
& $\begin{array}{c}\text { Recours } \\
\%\end{array}$ & Satisfaction & Recours & Satisfaction & Recours & Satisfaction \\
& $\%$ & $\%$ & $M$ & $\%$ & $M$ \\
Milieux scolaires & 55,6 & 6,2 & 33,3 & 6,4 & 41,7 & 6,2 \\
Hôpitaux & 44,4 & 5,0 & 6,7 & 9,0 & 37,5 & 5,8 \\
Centres d'écoute & 55,6 & 0,8 & 13,3 & 1,0 & 37,5 & 3,2 \\
Bureaux privés & 33,3 & 8,3 & 33,3 & 6,2 & 25,0 & 7,7 \\
Organismes communautaires & 11,1 & 8,0 & 20,0 & 7,0 & 25,0 & 7,8 \\
DPJ & 11,1 & 8,0 & 13,3 & 9,0 & 12,5 & 3,3 \\
CLSC & 11,1 & 7,0 & 26,7 & 6,3 & 8,3 & 8,0 \\
Centres de prévention suicide & - & - & 33,3 & 7,8 & 8,3 & 9,0 \\
Aucune ressource & - & - & 13,3 & - & 8,3 & -
\end{tabular}

a Minimum 0; maximum 10 


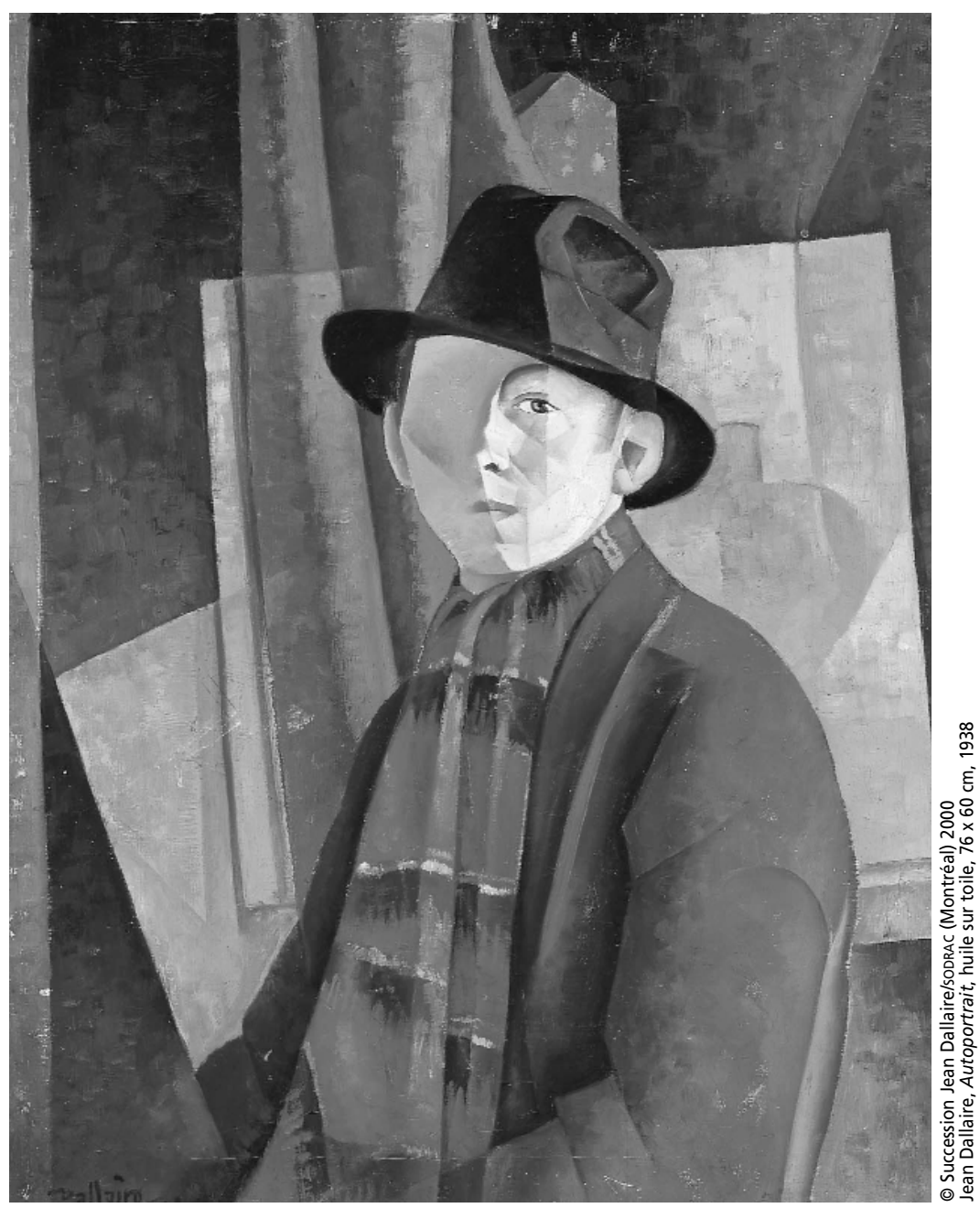

psychologique, plus il évalue négativement la ressource consultée dans le passé; et plus un jeune veut plaire à l'interviewer psychologue, plus il estime positivement la ressource utilisée avant sa dernière tentative de suicide.

Par ailleurs, nous avons repéré et abrégé différents commentaires repré- sentatifs de l'opinion des jeunes pour mieux comprendre leur appréciation des ressources. Lorsqu'il s'agit de la nature de l'aide reçue, si certains jeunes évaluent négativement diverses ressources, c'est que ces dernières ne répondent pas vraiment à leurs attentes. Cet écart entre les offres de service et les demandes des

Tableau 2

\section{Satisfaction liée aux trois ressources les plus utilisées selon la détresse} et la désirabilitéa

\begin{tabular}{l|c|c|c} 
Variables & $\begin{array}{c}\text { Milieux } \\
\text { scolaires } \\
\mathrm{n}=10\end{array}$ & $\begin{array}{c}\text { Hôpitaux } \\
\mathrm{n}=9\end{array}$ & $\begin{array}{c}\text { Centres } \\
\text { d'écoute } \\
\mathrm{n}=9\end{array}$ \\
$\begin{array}{l}\text { Niveau de détresse psychologique } \\
\quad \text { Élevé }\end{array}$ & 4,8 & 5,3 & 2,0 \\
$\quad$ Faible & 7,3 & 10,0 & 4,2 \\
Niveau de désirabilité sociale & 6,4 & 7,5 & 4,0 \\
$\quad$ Élevé & 5,3 & 4,4 & 1,7 \\
$\quad$ Faible & \\
a Le degré de satisfaction varie entre 0 minimum et 10 maximum.
\end{tabular}

jeunes se manifeste notamment dans l'évaluation que les jeunes effectuent de l'expertise et de l'attitude des intervenants. Le Tableau 3 vient illustrer ce que demandent les jeunes quand ils viennent solliciter de l'aide: la capacité d'accueil de leur souffrance et l'évaluation de cette souffrance apparaissent très importantes. Lorsque nous considérons le type de ressources utilisées dans la structure du réseau d'aide formelle, plusieurs remarques émergent pour chacune de ces ressources (voir le Tableau 4). Cela nous renvoie à nouveau au paradoxe entre les ressources «les plus utilisées» et les ressources «les moins appréciées». Se peut-il que ces jeunes, qui sont pour la plupart des suicidants récidivistes, suscitent, chez les intervenants qui les connaissent, deux réactions contradictoires qui rebutent les jeunes? Dans le premier cas, l'intervenant ressent une obligation d'agir de peur d'une récidive, et oublie d'écouter la souffrance du jeune. Cette attitude se retrouve plus souvent dans les milieux scolaires et hospitaliers utilisés par les jeunes de cette étude. Dans le deuxième cas, l'intervenant ne réussit pas à évaluer l'ampleur de la souffrance, parce que les moyens dont il dispose sont trop limités. Cette attitude se retrouve surtout au niveau des centres d'écoute et de la DPJ. Ainsi, nous suggérons qu'un intervenant, qui connaît un récidiviste, soit davantage tenté d'intervenir rapidement ou de ne pas accorder l'attention nécessaire à la gravité des problèmes du jeune.

\section{ENJEUX À CONSIDÉRER POUR INTERVENIR AUPRÈS DU JEUNE \\ SUICIDANT}

Les résultats de notre recherche nous amènent maintenant à la deuxième partie de notre exposé, dans laquelle nous traiterons des enjeux à considérer pour intervenir auprès des jeunes. Il est important d'interpréter ces éléments comme des pistes à approfondir et à valider éventuellement avec d'autres études.

\section{DES JEUNES AU BORD DU GOUFFRE}

Lorsque nous étudions attentivement le contexte de demande d'aide des jeunes, nous identifions un pattern qui découle logiquement de leurs histoires. Pour la plupart d'entre eux, lorsqu'un événement malheureux nouveau surgit, faisant écho à un traumatisme antérieur, il déclenche une montée d'angoisse importante et difficile à gérer. D'équilibre fragile, c'est-à-dire ne possédant pas le mécanisme d'adaptation adéquat pour contenir cette angoisse, les jeunes 
interviewés présentent une capacité limitée pour affronter cette pression émotive et situationnelle. C'est dans ce contexte, sur le point de "craquer», qu'ils cherchent un soutien pour les aider à contenir ou à altérer cette souffrance jugée à la limite du tolérable. Ces premiers éléments renseignent l'intervenant sur l'importance à accorder à l'intensité de l'angoisse et à la capacité d'endurance du jeune qui le consulte. Bien comprendre cette douleur et bien évaluer les mécanismes qui peuvent la contenir ou la réduire pourraient aider l'intervenant à prévenir le suicide. Reste le problème de la rapidité avec laquelle l'angoisse insupportable semble opérer. L'ensemble des jeunes interrogés expliquent avoir fait une tentative de suicide, même si certains intervenants avaient très bien abordé la question de leur douleur et de leur seuil de tolérance à cette douleur. La plupart des jeunes rapportent avoir été littéralement foudroyés par une angoisse insupportable qui les a mis dans un état "hors réalité» et les a poussés à commettre leur geste. Est-ce qu'une confirmation et une meilleure explication de ce processus chez le jeune suicidant pourraient favoriser de meilleures interventions auprès des jeunes?

\section{UN ESPOIR À CONSIDÉRER}

Dans un autre ordre d'idées, les résultats portant sur l'utilisation des services suggèrent qu'une proportion importante de jeunes rencontrés consulterait une ressource d'aide avant de faire leur dernière tentative de suicide. Cela laisse voir qu'il serait possible, avec une meilleure connaissance des soins à prodiguer et une meilleure concertation entre les services, d'arrêter le processus suicidaire. Les résultats indiquent également qu'une vaste gamme de services est utilisée, et ce, sans égard à leur spécificité. Il serait souhaitable, dans une étude ultérieure, de mieux saisir la contribution spécifique de chaque intervention en prévention du suicide. Chaque intervenant pourrait ainsi agir selon ses compétences et rediriger la demande d'aide au besoin. Reste enfin l'utilisation différente des ressources selon le sexe. Les répondants féminins semblent solliciter davantage une aide faisant appel à une structure d'accueil et d'écoute, tandis que les répondants masculins semblent recourir surtout à une aide de prise en charge. Il se peut, en raison de la socialisation du genre, que les femmes consultent plus au début du processus suicidaire que les hommes.

Tableau 3

Commentaires des jeunes à l'égard de l'aide reçue selon les composantes de la relation d'aide et l'évaluation de la suicidalité

\begin{tabular}{|c|c|c|}
\hline \multirow[t]{2}{*}{ Notions ${ }^{a}$} & \multicolumn{2}{|c|}{ Commentaires } \\
\hline & Favorables & Défavorables \\
\hline Respect & $\begin{array}{l}\text { II me prend tel que je suis } \\
\text { II va à mon rythme }\end{array}$ & $\begin{array}{l}\text { II me juge sans cesse } \\
\text { Il m'oblige à me confier }\end{array}$ \\
\hline Authenticité & $\begin{array}{l}\text { Il est «vrai» avec moi } \\
\text { Il dit ce qu'il pense }\end{array}$ & $\begin{array}{l}\text { Il est trop derrière son bureau } \\
\text { II me prend pour un numéro }\end{array}$ \\
\hline Présence & $\begin{array}{l}\text { II est là avec moi } \\
\text { II me sécurise }\end{array}$ & $\begin{array}{l}\text { Il est peu disponible et pressé } \\
\text { II m'accorde peu d'attention }\end{array}$ \\
\hline Écoute & $\begin{array}{l}\text { Il décode mes propos } \\
\text { II m'incite à parler }\end{array}$ & $\begin{array}{l}\text { II déforme tout mon vécu } \\
\text { II s'écoute parler }\end{array}$ \\
\hline Empathie & $\begin{array}{l}\text { Il comprend ma tristesse } \\
\text { Il s'adapte à moi }\end{array}$ & $\begin{array}{l}\text { II minimise mes problèmes } \\
\text { II y a trop de règles }\end{array}$ \\
\hline Responsabilité & $\begin{array}{l}\text { Il a confiance en moi } \\
\text { II me redonne espoir }\end{array}$ & $\begin{array}{l}\text { II me sous-estime } \\
\text { II veut trop pour moi }\end{array}$ \\
\hline Évaluation critérielle & $\begin{array}{l}\text { II prend des informations } \\
\text { liées à mon état dépressif }\end{array}$ & $\begin{array}{l}\text { Il m'interroge et m'accole } \\
\text { des étiquettes }\end{array}$ \\
\hline Évaluation fonctionnelle & $\begin{array}{l}\text { Il avance l'hypothèse } \\
\text { d'un déficit relationnel }\end{array}$ & $\begin{array}{l}\text { II n'arrive pas à expliquer } \\
\text { mon état et ma situation }\end{array}$ \\
\hline
\end{tabular}

a Respect: capacité de reconnaître la dignité et la valeur de l'autre. Authenticité: capacité d'être soi-même, en toute sincérité, dans la relation avec l'autre. Présence: capacité de se centrer sur l'autre en l'accompagnant de façon efficace tant psychologiquement que physiquement. Écoute: capacité de capter et de comprendre les messages verbaux et non verbaux de l'autre. Empathie: capacité de se mettre à la place de l'autre. Responsabilité: croire au rôle actif de l'individu dans ses possibilités de changements. Évaluation critérielle: procédé menant à un diagnostic psychiatrique. Évaluation fonctionnelle: procédé visant à cerner les facteurs prédisposants, précipitants et de maintien d'un trouble.

Tableau 4

Commentaires des jeunes à l'égard de chacune des ressources

\begin{tabular}{l|l} 
Ressources & Commentaires \\
Milieux scolaires & Aide réconfortante, mais trop interventionniste \\
Hôpitaux & Aide mobilisante, mais trop structurante \\
Centres d'écoute & Aide ponctuelle, mais trop générale \\
Bureaux privés & Aide disponible et compréhensive \\
Organismes communautaires & Aide de prise en charge spontanée \\
DPJ & Aide sécuritaire, mais trop administrative \\
CLSC & $\begin{array}{l}\text { Aide vivifiante, mais parfois rapide } \\
\text { (nombre limité de rencontres) }\end{array}$ \\
Centres de prévention suicide & Aide soutenante et concrète
\end{tabular}

\section{UNE APPROCHE PERSONNALISÉE}

ET CIRCONSCRITE À PRIVILÉGIER

Les résultats liés à la satisfaction des services employés sont probablement les plus intéressants pour l'intervenant. Ce que les jeunes retiennent de l'aide obtenue, c'est la manière dont l'aidant reçoit et évalue la demande d'aide. Bien que la réponse vis-à-vis d'un jeune signalant son intention de se tuer soit différente en fonction de la situation (urgence, relation face à face, aide téléphonique, etc.), la façon d'aborder la demande d'aide du jeune demeure la même, d'un contexte à l'autre. En tout premier lieu, l'intervenant doit considérer le jeune comme une personne, comme un être humain en mal de vivre, et qui cherche une voie pour exprimer sa souffrance. C'est grâce à une attention personnalisée qu'une relation de confiance s'installe entre l'intervenant et le jeune à aider. Et c'est à partir de cette confiance que le processus de dévoilement et de confidence s'enclenche. Par ailleurs, l'accueil et l'écoute empathique ne semblent pas suffisants pour bien saisir la demande d'aide du jeune suicidant. L'intervenant doit évaluer cette demande d'aide en la replaçant dans l'ensemble du fonctionnement du jeune, afin de repérer ce qui favorise un mode de réponse par l'agir 
suicidaire. Cette évaluation de la suicidalité comporte quatre niveaux. Pour les besoins de l'évaluation, et afin de faciliter la communication entre les intervenants, il est nécessaire, en premier lieu, de se référer à une classification. Les conduites suicidaires se classifient habituellement en quatre catégories: l'idéation, la verbalisation de l'intention, la tentative et le suicide complété. À un deuxième niveau, la problématique suicidaire s'évalue en termes de manifestation d'une perturbation psychologique et/ou psychiatrique. Avant d'offrir une aide quelconque, l'intervenant devra d'abord être en mesure d'identifier de telles manifestations. En troisième lieu, l'intervenant a intérêt à tenir compte des facteurs de risque de suicide (p. ex., abus de drogue) chez le jeune qui le consulte. Ces éléments contribueront à cerner la dangerosité de la situation. Enfin, l'évaluation doit porter sur une exploration personnelle et profonde de la situation concrète qui amène le jeune à vouloir s'enlever la vie.

\section{DES ATTITUDES PLUS AUTOCRITIQUES}

\section{À FAVORISER CHEZ LES INTERVENANTS}

Enfin, si nous poussons plus loin notre analyse des commentaires émis par les jeunes relativement à l'aide reçue (Tableau 3), il appert que les intervenants devraient faire preuve de plus d'autocritique vis-à-vis de la relation d'aide qu'ils mettent en place, et s'efforcer de mieux comprendre la psychosociologie des jeunes de notre culture. Vouloir sans cesse intervenir, et vouloir intégrer les jeunes dans les rangs de la société, risquent d'accroître encore plus leur angoisse. Ainsi, paradoxalement, l'aide mal prodiguée peut devenir un facteur de risque de suicide.

\section{UN TRAVAIL SUPPLÉMENTAIRE À FAIRE POUR PRÉVENIR LE SUICIDE}

Cette réflexion repose sur des données issues d'une étude exploratoire dont les principales limites sont la représentativité et la taille de l'échantillon, ainsi que les caractéristiques psychologiques des sujets interviewés. Malgré ces faiblesses, ces données nous aident à réaffirmer l'importance à accorder à la formation des intervenants et à l'arrimage entre l'aide sollicitée et le mode d'aide à dispenser. Nous sommes persuadés de la nécessité de mieux former et de mieux équiper les intervenants, afin que ces derniers puissent offrir des soins plus appropriés et plus intégrés à la réalité des jeunes vivant des expériences suicidaires. À cet égard, le gouvernement du Québec proposait, dans sa Stratégie qué- bécoise d'action face au suicide, un objectif en ce sens: assurer la coordination et la continuité des services à offrir aux suicidants, tout en favorisant l'amélioration des compétences des professionnels dans le domaine. Espérons que, dans un avenir rapproché, cet objectif se traduira par des actions concrètes dans la communauté.

\section{Notes}

1 Cet article est basé sur une recherche qui a été soutenue par une subvention du Secrétariat à la jeunesse, et reconnue par la direction scientifique du Fonds de recherche en santé du Québec. Les auteurs remercient Luce Des Aulniers pour sa révision du manuscrit, ses commentaires et ses suggestions.

2 G. DULAC, Les demandes d'aide des hommes, Rapport de recherche du Groupe d'études appliquées sur la famille de l'Université McGill, Montréal, 1997, 38 pages.

3 Un «suicidant» est un individu qui a déjà fait une tentative de suicide. Le terme «suicidé» est utilisé pour désigner une personne qui est morte par suicide. Et un «suicidaire» représente une personne qui pense sérieusement à s'enlever la vie, mais qui ne passe pas à l'acte.

4 J.P. LAVOIE et al., Réseau social, recherche d'aide et soutien social chez une population de parasuicidaires francophones âgés de 18 à 64 ans. Rapport de recherche de l'Unité de recherche psychosociale de l'Hôpital Douglas, Montréal, 1990, 104 pages.

5 A.E. GROSS et P.A. McMULLEN, «Models of Help-Seeking Process», dans New Direction in Helping, sous la direction de A. NADER, J.D. FISHER et B.M. De PAULO, New York, Plenum Press, 1983, p. 45-70.

6 Ce document, produit en 1998 par le ministère de la Santé et des Services sociaux, énonce des objectifs de mise en œuvre d'actions pour prévenir le suicide.

7 Le Conseil permanent de la jeunesse (CPJ) est un organisme consultatif créé en vertu d'une loi votée en 1987 par l'Assemblée nationale. Il a notamment pour mission de procéder à la réalisation de travaux pouvant éclairer les divers ministères, et leurs partenaires, sur différents dossiers importants liés à la jeunesse. C'est dans le cadre de la préparation d'un Avis sur la question de la prévention du suicide chez les jeunes que le CPJ souhaitait enrichir son document d'une étude exploratoire portant sur la demande d'aide formelle de jeunes suicidants québécois.

8 Il s'agit d'une étude exploratoire en ce sens qu'il est davantage question de construire une problématique que de vérifier empiriquement des faits.

9 Premièrement, les chercheurs ont recruté des participants en provenance de certains hôpitaux psychiatriques, de centres de prévention suicide, d'organismes communautaires et d'institutions d'enseignement ou qui avaient répondu à des annonces parues dans les journaux. Deuxièmement, les chercheurs ont divisé en deux temps la réalisation des entre- vues. La première consistait en la préparation de l'entretien, l'autre à l'entrevue avec supervision.

Troisièmement, une psychologue a mené une entrevue semi-structurée enregistrée sur bande audio; à la fin de l'entrevue, les jeunes ont répondu à deux questionnaires (l'un sur l'échelle de détresse psychologique [IDPESQ-14], l'autre sur l'échelle de désirabilité sociale [Mc SDS-Form C]). Quatrièmement, les chercheurs ont pris connaissance de l'ensemble des données sur papier et sur bande audio, en compilant celles qui étaient numériques et en dépouillant celles qui étaient sémantiques. Cinquièmement, les chercheurs ont procédé à une synthèse des résultats selon le but de l'étude.

10 Cette technique consiste à découper le discours d'une personne en plusieurs énoncés significatifs, à réduire ces énoncés en mots clés et à réorganiser l'ensemble du matériel selon ces catégories. Voir L. L'ÉCUYER «L'analyse de contenu: notion et étapes» dans Les méthodes de la recherche qualitative, sous la direction de J.-P. DESLAURIERS, Sillery, Presses de l'Université du Québec, 1987, p. 49-65.

11 La tentative de suicide devait avoir eu lieu au cours de l'année précédant le début de la recherche. Lorsque nous évaluons la gravité de la dernière tentative de suicide en termes de létalité (risque mortel du geste en raison du caractère irréversible de la méthode et de la faible possibilité de sauvetage), nous obtenons le portrait suivant: $58 \%$ des répondants ont posé un geste de létalité faible (mort improbable); $13 \%$ un geste de létalité moyenne (mort plus ou moins probable) et $29 \%$ un geste de létalité élevée (mort probable à moins d'une intervention médicale). Ces estimations effectuées par les deux chercheurs sont basées sur une grille connue. Voir L. SAIT, R.W. CONROY et B.D. EHLER "Lethality of Suicide Attempt Rating Scale», Suicide and Life-Threatening Behavior, vol. 14, no 4, 1984, p. 215-243.

12 Nous avons rencontré, dans leur milieu, des jeunes du Bas-Saint-Laurent, du Saguenay-Lac-St-Jean, de Québec, de la Mauricie-Bois-Francs, de Montréal, de l'Outaouais, de l'Abitibi-Témiscamingue, de Chaudière-Appalaches, de Laval, de Lanaudière et de la Montérégie.

13 Plus de $65 \%$ des jeunes rapportent avoir eu des histoires d'abus physique/sexuel ou des histoires de toxicomanie/rejet. Dans le premier cas, il s'agit surtout de jeunes femmes, dans le second cas, de jeunes hommes.

14 Ainsi, parmi les 24 jeunes, 15 ont fait déjà une première tentative de suicide avant leur dernière tentative de suicide.

15 Ces nouveaux problèmes avaient trait dans plus de $50 \%$ des cas à des pertes d'estime de soi ou à des déceptions amoureuses.

16 Pour chaque ressource utilisée, le répondant évaluait sur une échelle de type Likert variant de 0 (pas du tout satisfait) à 10 (entièrement satisfait) son niveau d'appréciation. 